\title{
Fusion of photogrammetry and coherence scanning interferometry data for all-optical coordinate measurement
}

\author{
Richard Leach (2)a, Danny Sims-Waterhouse ${ }^{a}$, Fabrizio Medeossib, Enrico Savio (1) ${ }^{\mathrm{b}}$, Simone Carmignato (2) ${ }^{\mathrm{c}}$, \\ Rong Sua
}

a Manufacturing Metrology Team, Faculty of Engineering, University of Nottingham, Nottingham NG8 1BB, UK
${ }^{b}$ Department of Industrial Engineering, University of Padua, via Venezia 1, 35131 Padova, Italy
${ }^{c}$ Department of Management and Engineering, University of Padua, Stradella San Nicola 3, 36100 Vicenza, Italy

Multisensor data fusion is an approach to enlarge the potential applicability of measuring techniques and improve accuracy, taking advantage of the strengths of different techniques. In this work, we present a new method for the fusion of photogrammetry and coherence scanning interferometry (CSI) data. This method allows the photogrammetry data to be accurately scaled with reference to the CSI data, and in turn the exact locations of multiple CSI measurements can be determined in the coordinate system defined by photogrammetry. The culmination of this work is to allow for high-accuracy threedimensional optical coordinate measurement and surface topography measurement simultaneously.

Data fusion, surface topography, interferometry, photogrammetry, coordinate measurement

\section{Introduction}

Multisensor data fusion is a promising approach to enlarge the spatial bandwidth of measuring techniques and/or improve accuracy, taking advantage of the strengths of different techniques. The 2009 CIRP Keynote [1] provides excellent insight into the use of multisensor data fusion for dimensional metrology in order to get holistic, more accurate and complete information about a workpiece based on measurement from one or more sensors, data processing and modelling of the measurement procedure [2]. Generally, data types are indexed as homogeneous integration when combining the same type of data (e.g. 3D point clouds), while inhomogeneous integration is required in all other cases. Different methods have been developed for the fusion of information from different sensors. A two-stage multisensor data fusion model (Gaussian model) is presented by Colosimo et al. [3]. Fringe projection data (high density points but low accuracy) are fused with a smaller set of data acquired with a touch-probe coordinate measuring machine (CMM) (lower point density but higher accuracy). The Gaussian process model is built using the highdensity dataset, then the two datasets (homogeneous datasets) are linked through a linkage model. In reference [4], the Gaussian process method for measurement of complex geometries is presented and the validity and application of the method is evaluated. Moreover, the same approach is applied by Chen [5]; changing the systems and using artificial datasets (fusion of laser scanning microscopy and atomic force microscopy). Different methods, based on weighted fusion functions, are described elsewhere [6,7]. All the previously described methods only work effectively for homogeneous datasets.

Ramasamy and Raja [8] compared different data fusion algorithms: coherence scanning interferometry (CSI) data were fused using three different methods. Regional energy based, regional edge intensity based, and a combination of wavelet coefficients and local gradients were applied on different samples. Regional edge intensity was selected as the preferred fusion method for surface metrology, considering that the method was mainly focused on homogeneous datasets (CSI data at different magnifications). A multi-scale approach was presented and discussed both for the fine registration and for the fusion. A more general study was developed in reference [9], in which inhomogeneous datasets are fused: from an optical CMM and CSI.

In this paper, a registration method and a fusion of data from different domains (inhomogeneous datasets), moreover from completely different scales is presented. The two methods are photogrammetry (data as a 3D point cloud) and CSI (data as a series of heights on a uniform 2D grid). The higher resolution component of the photogrammetry data is the intensity information rather than the 3D shape of the measured sample. The previous work outlined in this paper [3-9] was mainly focused on the fusion of surface topography measurements over similar measurement areas, using different measurement methods. However, in this work we attempt to register data on completely different scales, focusing on the localisation of micro-scale surface texture with respect to macro-scale form information.

\section{Methods}

CSI is a measurement technique typically used for the measurement of micro-features and surface texture [10]. The field of view (FOV) for CSI methods typically ranges from tens of micrometres to a few millimetres, requiring stitched measurements to cover larger areas. Surface topography measurement within a single FOV for CSI has relatively high accuracy compared to stitching of a large number of single measurements. Stitching of multiple CSI measurements can result in significant error of the surface form, due to the increased measurement uncertainty from the complex form and large roughness of the surface [11] and as a result of the lateral distortion of the CSI system [12].

Photogrammetry is a passive triangulation based method, by which many images of a sample are used to produce a point cloud of the surface [13]. The point cloud is produced through the triangulation of many corresponding points over the surface of the sample and can capture a measurement of the sample's 3D 
geometry. As photogrammetry relies on only images to reconstruct form information, the FOV that can be achieved covers a large range of values, e.g. from $10 \mathrm{~mm}$ to $100 \mathrm{~km}$. At a FOV of around $10 \mathrm{~mm}$ to $20 \mathrm{~mm}$, photogrammetry can achieve spatial resolutions on the order of $4 \mu \mathrm{m}$ to $10 \mu \mathrm{m}$, depending of the camera being used. Recent work has also shown that photogrammetry is able to obtain measurement uncertainties on the order of $10 \mu \mathrm{m}$ over a FOV of around $20 \mathrm{~mm}$ [14], although accurate scaling methods over such ranges is problematic. Photogrammetry is able to capture form information at a lower spatial resolution than CSI with arbitrary scale but can do so over a much larger FOV.

Despite the lower spatial resolution of the form information captured by photogrammetry, the intensity information provides much higher resolution information about the object's surface. It is the intensity information of the photogrammetry data that provides sufficient overlap in sampled spatial frequencies that allows pre-alignment of the CSI point cloud to be achieved. The pre-alignment is required in order to account for the significant differences in the geometries measured by the CSI and photogrammetry. As there is a significant difference in the spatial bandwidths captured by the photogrammetry and CSI measurement methods, the direct application of iterative closest point (ICP) algorithms to the data will not result in a successful registration [6]. The nature of photogrammetry also means the resultant point cloud will have an arbitrary scale, requiring additional calibration to extract metric information. Fortunately, by pre-aligning the photogrammetry data with the CSI data, the photogrammetry point cloud can be appropriately scaled prior to the final registration.

The general pipeline for the fusion process can be shown in Figure 1. Initially, the point cloud from the photogrammetry system is sampled as a 2D image and the CSI intensity map is processed in order to better represent the visual appearance of the region being measured. Feature matching algorithms are applied to the photogrammetry and CSI images in order to generate a planar transformation and scaling between the two datasets. This planar transform and scale factor allows the photogrammetry scale to be determined and the CSI data to be aligned in a single plane. This planar alignment of the CSI data fixes three degrees of freedom, ensuring an ICP algorithm will successfully minimise the point cloud differences in an acceptable time frame. Finally, the point clouds are fused by removing points in the photogrammetry data that overlap with the CSI measurement region. The CSI data is then merged with the photogrammetry point cloud, replacing the original low-resolution data.

For this application, the CSI data were acquired with a ZYGO NewView $^{\mathrm{TM}} 8300$ CSI system using a Michelson $5.5 \times$ objective lens and a $0.5 \times$ zoom lens. The images used for the photogrammetry were acquired with a Nikon D3300 DSLR with a $60 \mathrm{~mm}$ macro lens, combining a total of thirty images and covering a FOV of approximately $(20 \times 20) \mathrm{mm}$. The reconstruction of the images was achieved with Agisoft PhotoScan [15] and the final data was exported as a tab-delimited text file (the point cloud).

\section{Results}

The first stage of the pipeline (shown in Figure 1) requires both datasets to be projected onto a 2D image for feature recognition methods to be applied. The nature of the CSI measurement means the CSI intensity map is already in the correct format. However, the photogrammetry data must be projected on to a plane and sampled into a discrete image. For this work, a one pence coin was used to demonstrate the procedure. For the coin sample, the point cloud was fitted to the $x-y$ plane and projected along the $z$-axis to produce an image. The intensity image of the photogrammetry data is generated by sampling the RGB data of the point cloud in the $x-y$ plane and then converting to a grey-scale image. The sampling frequency used to generate the image was chosen to approximately match the number of points in the point cloud. Assuming a square image, the number of samples was calculated as the square root of the total number of points to maximise the spatial resolution of the intensity image.

Once the intensity images for both the CSI and photogrammetry data have been generated, simple image processing is applied to the CSI intensity map to increase the contrast of any features in the image.

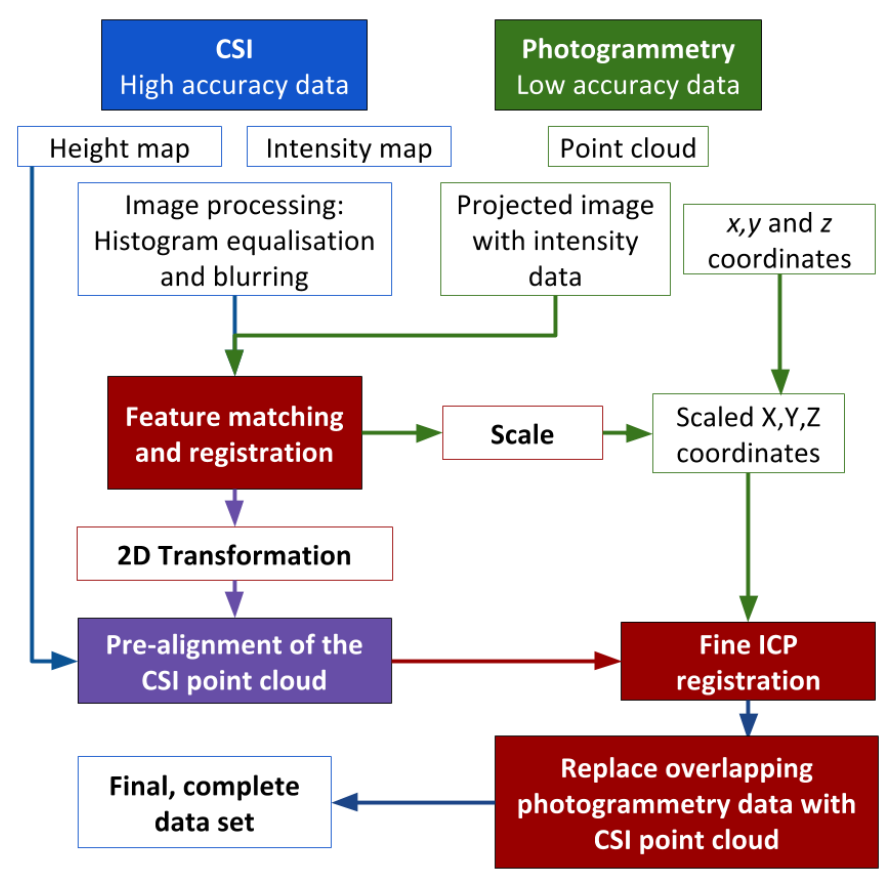

Figure 1. Pipeline for the fusion of the CSI and photogrammetry data

Prior to the feature detection process, the CSI data is further processed in order to account for the high number of high spatial frequency features visible in the intensity images. A Gaussian filter is applied to the CSI intensity image effectively blurring highfrequency features, such that only features visible in both datasets are detected. Effectively, the spatial bandwidth of the CSI data is reduced in order to only leave the region overlapping with the photogrammetry data.

With two images for comparison, image feature recognition algorithms can now be applied to determine the corresponding points between the datasets. Due to their performance under scale, rotation and illumination differences, scale-invariant feature transform (SIFT) algorithms where used to find corresponding features between the images. Once the SIFT features have been detected in both images and the descriptor vectors are assigned, the features are matched, and a set of corresponding pixel coordinates are produced [16]. In order to remove any falsepositive matches, a fundamental matrix is calculated for the matched image coordinates and any points that do not satisfy the matrix are removed. As the fundamental matrix describes the relationship between points under different views, this removes false-positive matches that do not follow the general trend in point translation.

Finally, the relative rotation, translation and scale factor between the remaining point correspondences can be calculated. This will provide further refinement of the data points until only positive matches remain. The translation matrix between the two datasets is described by the equation 


$$
\left[\begin{array}{c}
x_{C S I} \\
y_{C S I} \\
1
\end{array}\right]=T\left[\begin{array}{c}
x_{C S I}^{\prime} \\
y_{C S I}^{\prime} \\
1
\end{array}\right]
$$

where $T$ is a $2 \mathrm{D}$ rigid homogenous transform, $\left(x_{C S I}^{\prime}, y_{C S I}^{\prime}\right)$ are the old CSI coordinates and $\left(x_{C S I}, y_{C S I}\right)$ are the shifted CSI data points. The 2D homogenous transform matrix encodes the relative translation and rotation to align the CSI data in the $x$ - and $y$-axes. The scale information is inversely applied to the photogrammetry point cloud in order to scale the data. In the case of multiple CSI measurements, an average scale can be calculated and applied to the data post-registration.

With the application of the 2D transformation matrix shown above, the CSI data is now registered in the $x-y$ plane. This 2D registration is shown in Figure 2, where the intensity image of the CSI and photogrammetry measurements are shown.

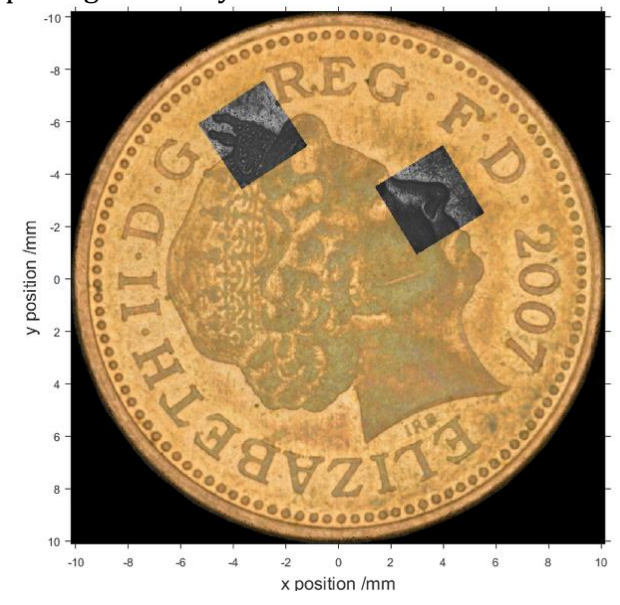

Figure 2. CSI data alignment in the $x-y$ plane

A simple $z$-axis registration is then applied by shifting the mean $z$ heights of both datasets to zero. With the CSI data now fixed in three degrees of freedom, ICP algorithms can be applied to the CSI and photogrammetry point clouds in order to refine the $z$-axis translation and rotations about the $x$ - and $y$-axes. The preliminary alignment is clearly necessary in order to reduce the number of iterations required in the ICP algorithm and to ensure a convergence of the ICP algorithm in a reasonable amount of time (the degrees of freedom are reduced from six to three thanks to the preliminary phases).

After the registration, the data are fused in to a single point cloud. The area of the photogrammetry point cloud corresponding to the CSI registered image is removed and the CSI data substituted. The final point cloud is shown around the CSI measurement regions in Figure 3 and Figure 4 . The whole process is completed within around three to four minutes.

As expected, discontinuities are observed in the fused point cloud around sharp transitions in height. The main difference in the CSI and photogrammetry point clouds is not the heights of any particular region, but the distinction of where the step in height appears. This improvement in the localisation of micro-scale features demonstrates the success of the method in increasing the bandwidth of the fused point cloud.

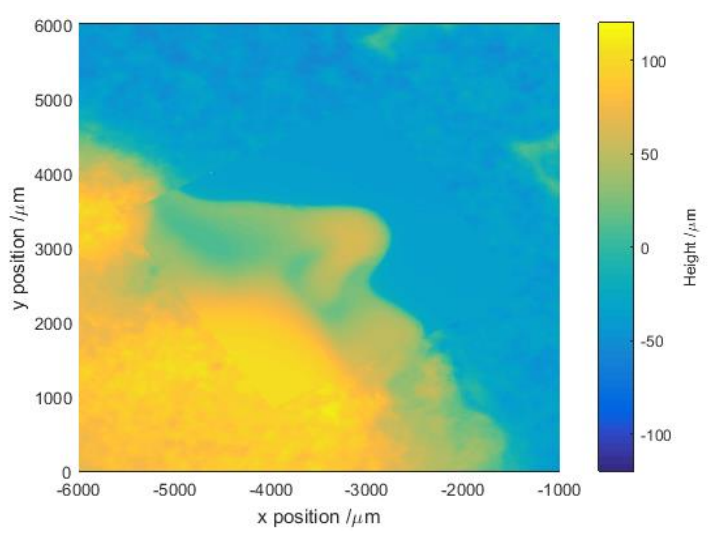

Figure 3. Height map of the fused photogrammetry and CSI point clouds in the nose measurement region

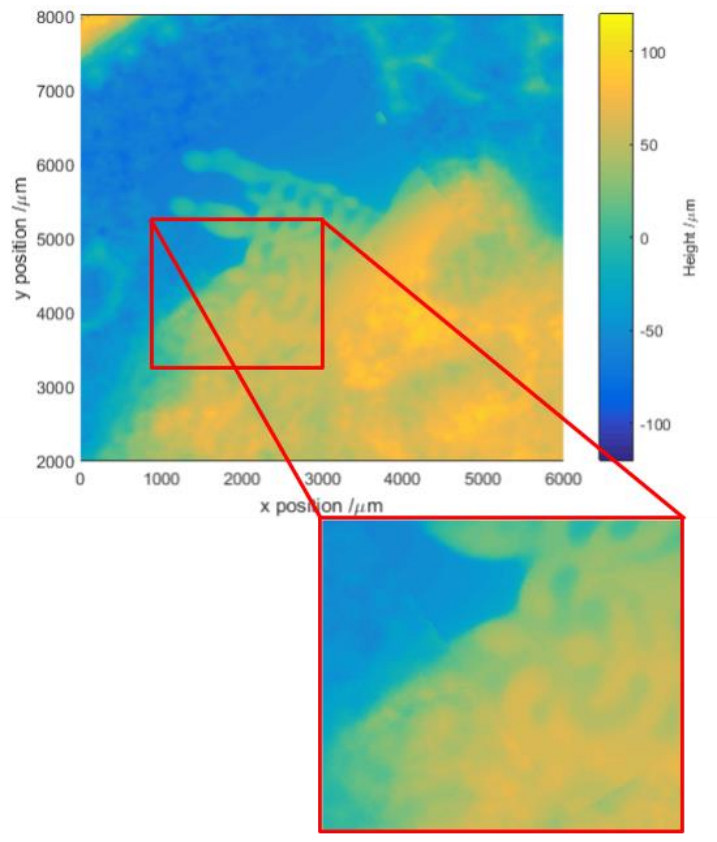

Figure 4. Height map of the fused photogrammetry and CSI point clouds in the crown measurement region with magnified region

With the CSI and photogrammetry data now registered, the topographies can be compared in order to determine the effectiveness of the registration procedure. The topography differences can be seen in Figure 5 and Figure 6 for the nose and crown measurement, respectively.

As can be seen in Figures 5 and 6, the topographies generally agree over the majority of the surface to less than $10 \mu \mathrm{m}$. As would be expected, the most significant differences are observed on high spatial frequency features, such as edge responses and finer details not observed in the photogrammetry point cloud. These results confirm that the final point cloud presents an increased resolution and accuracy in the areas measured by the CSI (small details), and the overall and large-scale information from the photogrammetry. Additionally, the entire coin surface is measured by stitching 5by- 5 CSI measurements. The distance between the centre-of-mass of the two CSI measurements (as shown in Figure 2) was found to be $7.582 \mathrm{~mm}$ based on the stitching result. The same distance in the fused data was found to be $7.690 \mathrm{~mm}$. 


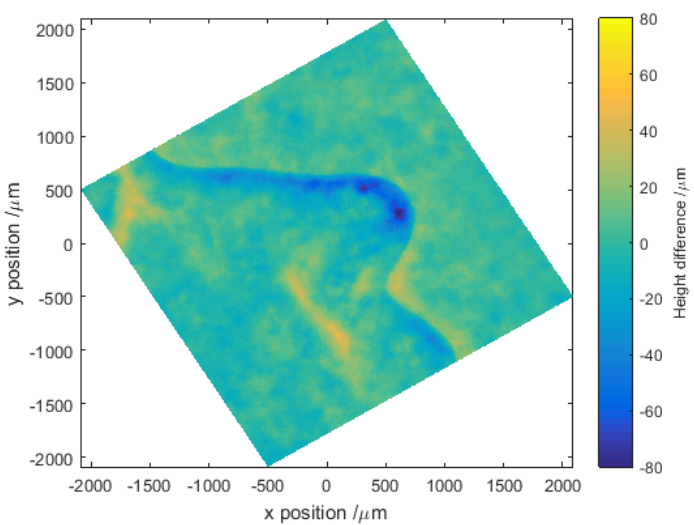

Figure 5. Difference map between the photogrammetry and CSI point cloud data at the nose measurement region

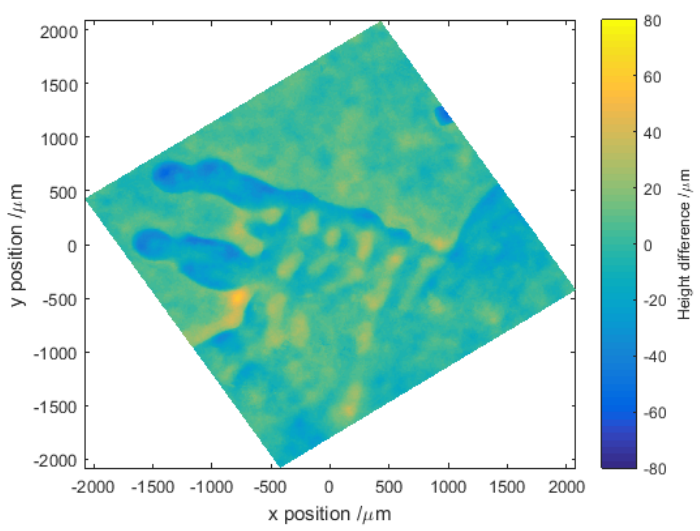

Figure 6. Difference map between the photogrammetry and CSI point cloud data at the crown measurement region

\section{Discussion}

A comparison of the final point clouds gives a difference of $1.4 \%$ between the fusion data and stitching measurement. Considering the minimal overlap in spatial passbands between CSI and photogrammetry, significant differences in small-scale features, corresponding to high spatial frequencies, is to be expected. This difference will significantly degrade the accuracy of the image and point cloud registration and result in the scaling error that influences the lateral distance measurement. However, flatter regions, corresponding to low spatial frequencies, have height differences of under $10 \mu \mathrm{m}$, in agreement with measurement uncertainties typically achieved by photogrammetry systems [14]. The fused point cloud covers features on the order of $4 \mu \mathrm{m}$ to 20 $\mathrm{mm}$, allowing form and micro-scale texture measurement simultaneously.

Considering the nature of this fusion method, significant differences in surface height are expected and demonstrate the additional surface texture information gained. As can be seen in Figure 3 and Figure 4, there is a general agreement in the aligned datasets, with differences mainly occurring around edges and small-scale features. Figure 5 and Figure 6 further demonstrate the success of the registration with flatter regions at different heights exhibiting similar levels of differences.

The absolute accuracy of the data fusion technique is out of the scope of this paper but will be evaluated in future work through the measurement of a pre-calibrated geometry. Key areas of work will be on the improvement of the image registration process and increasing the number of features that are detected, and the development of a robust point cloud registration algorithm for handling the minimal overlap in spatial passband between different measuring techniques.

The fused point cloud presents a substantially increased spatial bandwidth of the surface measurement. A well-documented example of a high-value product that would benefit from the use of this method is the blades used for gas turbine engines. The blades are typically freeform geometries that vary in long-axis size from a few tens of centimetres to metres. Aside from their difficult-tomeasure freeform geometries, turbine blades have stringent surface texture requirements and can have sub-millimetre surface features for cooling (high aspect ratio cooling holes). Typically, the form and texture of the blades would be measured with different instruments, therefore, requiring complex alignment and registration processes due to the different coordinate frames used by each instrument. Full digital representation of the blade is required for modelling and later assembly operations. All these issues can be solved by the using the multi-scale fusion to produce a digital twin of the object plus its tolerance profiles.

\section{Acknowledgements}

This work was supported by the Engineering and Physical Sciences Research Council (EPSRC) (Grant Nos. EP/ M008983/1 and EP/L016567/1); European Metrology Programme for Innovation and Research (EMPIR) project MetHPM (14IND09). EMPIR is jointly funded by the EMPIR participating countries within EURAMET and the European Union.

\section{References}

[1] Weckenmann, A., Jiang, X., Sommer, K. D., Neuschaefer-Rube, U., Seewig, J., Shaw, L., Estler, L., 2009, Multisensor data fusion in dimensional metrology, Ann. CIRP, 58/2:701-721.

[2] Ghiotti, A., Schöch, A., Salvadori, A., Carmignato, S., Savio, E., 2015, Enhancing the accuracy of high-speed laser triangulation measurement of freeform parts at elevated temperature, Ann. CIRP, 64/1:499-502.

[3] Colosimo, B. M., Pacella, M., Senin, N., 2015, Multisensor data fusion via Gaussian process models for dimensional and geometric verification, Prec. Eng., 40:199-213.

[4] Yin, Y., Ren, M. J., Sun, L., Kong, L., 2016, Gaussian process based multiscale modelling for precision measurement of complex surfaces, Ann. CIRP, 65/1:487-490.

[5] Chen, Y., 2016, Data fusion for accurate microscopic rough surface metrology, Ultramicroscopy, 165:15-25.

[6] Wang, J., Leach, R. K., Jiang, X., 2015, Review of the mathematical foundations of data fusion techniques in surface metrology, Surf. Topog. Metr. Prop., 3/2.

[7] Wang J, Pagani, L, Leach, R. K, Zeng, W Colosimo, B. M., Zhou, L., 2017 Study of weighted fusion methods for the measurement of surface geometry, Prec. Eng., 47:111-121.

[8] Ramasamy, S. K., Raja, J., 2013, Performance evaluation of multi-scale data fusion methods for surface metrology domain, J. Manufac. Sys., 32/4:514522.

[9] Ramasamy, 2011, Multi-scale data fusion for surface metrology. Proquest Dissertation Publishing

[10] P. de Groot, 2011, Coherence scanning interferometry. In: Leach, R. K. Optical measurement of surface topography, 1st ed. Springer-Verlag Berlin Heidelberg.

[11] Su, R., Wang, Y., Coupland, J., Leach, R., 2017, On tilt and curvature dependent errors and the calibration of coherence scanning interferometry, Opt. Express, 25:3297-3310.

[12] Ekberg, P., Su, R., Leach, R., 2017, High-precision lateral distortion measurement and correction in coherence scanning interferometry using an arbitrary surface, Opt. Express, 25:18703.

[13] Luhmann, T., Robson, S., 2011, Close range photogrammetry: Principles, techniques and applications. Whittles Publishing.

[14] Sims-Waterhouse, D., Piano, S., Leach, R., 2017, Verification of micro-scale photogrammetry for smooth three-dimensional object measurement, Meas. Sci. Technol., 28/5.

[15] Agisoft, 2017, Agisoft PhotoScan. Agisoft.

[16] Lowe, D. G., 2004, Distinctive image features from scale-invariant keypoints, Int. J. Comp. Vis., 60/2:91-110. 